\title{
Monitoramento da carga interna \\ de um período de treinamento em jogadores de voleibol
}

CDD. 20.ed. 796.073

796.325

http://dx.doi.org/10.1590/1807-55092015000100005

\author{
Victor Hugo de FREITAS* \\ Bernardo MILOSKI** \\ Maurício Gattás BARA FILHO***
}

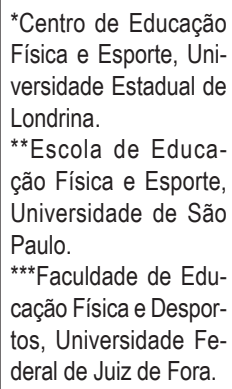

*Centro de Educação Física e Esporte, Universidade Estadual de Londrina.

**Escola de Educação Física e Esporte, Universidade de São Paulo.

${ }^{* *}$ Faculdade de Educação Física e Desportos, Universidade Federal de Juiz de Fora

0 objetivo desse estudo foi monitorar e analisar a dinâmica da carga interna, a partir do método da Percepção Subjetiva de Esforço da sessão (PSE-sessão), durante um periodo de treinamento no voleibol. Doze jogadores de uma equipe de voleibol $(23,5 \pm 3,4$ anos, 88,2 $\pm 11,4 \mathrm{~kg}, 190,4 \pm 10,8 \mathrm{~cm})$ foram monitorados durante 22 semanas de treinamento divididas em cinco mesociclos. A ANOVA revelou haver diferenças significativas nos valores de carga de treinamento semanal total (CTST), monotonia e "strain" entre os mesociclos $(p<0,05)$. A CTST do mesociclo 1 foi menor quando comparada aos mesociclos 2 , 4 e 5. Os valores de monotonia foram baixos, com valores inferiores a 1,4 0,1 UA. 0 coeficiente de variação da média da CTST das 22 semanas foi de $16 \%$. 0 valor médio da média da CTST das 22 semanas foi de 1790,9 UA. 0 menor valor de média da CTST das 22 semanas foi de 1324,3 UA e o maior valor foi de 2232,7 UA. Desse modo, a carga interna de treinamento apresentou momentos de maior e menor magnitude entre as semanas, e aumentou do primeiro para o segundo mesociclo com manutenção das mesmas nos mesociclos seguintes.

Palavras-chave: Esporte; Educação física; Treinamento; Desempenho.

\section{Introdução}

O voleibol é um esporte intermitente, com açôes de alta intensidade e curta duração, intercaladas por momentos de baixa intensidade ${ }^{1}$. A superioridade física, técnica e tática apresentada por equipes mais bem sucedidas com vitórias em jogos ${ }^{2}$ sugerem a necessidade de um processo de treinamento eficiente para se alcançar um elevado nível competitivo.

O sucesso do treinamento, por sua vez, depende do equilíbrio entre a magnitude da carga de treinamento e a recuperação aplicada ${ }^{3-4}$. Por exemplo, Nesser e Demchack ${ }^{5}$ mostraram que uma prétemporada com 24 sessóes de treinos em quadra, 12 sessôes de treinamento resistido e dois dias de descanso dentro de um período de 14 dias acarretou em queda significativa da altura alcançada no salto vertical de ataque $(-3,3 \mathrm{~cm})$, de bloqueio $(-7,7$ $\mathrm{cm})$ e no teste de agilidade $(+0,21 \mathrm{~s})$ em jogadoras de voleibol da primeira divisão da National Collegiate Athletic Association (NCAA). Além da queda do desempenho, das 13 atletas que participaram da pré-temporada, cinco se lesionaram durante a temporada competitiva. Por outro lado, Nesser e Dемснаск ${ }^{5}$ reportaram no mesmo estudo que na pré-temporada seguinte, quando as sessóes de treinamento foram reduzidas para 17 sessóes de treinos em quadra, 10 sessóes de treinamento resistido e um dia de descanso dentro de um período de 14 dias, não foi observada queda do desempenho nos testes de salto vertical de ataque, de salto vertical de bloqueio e de agilidade, e nenhuma atleta se lesionou durante a temporada competitiva. Nesse sentido, o constante monitoramento das cargas de treinamento é essencial para direcionar adequadamente a magnitude das cargas e tipos de treinamento aplicados.

Em esportes coletivos como o voleibol, muitas vezes o mesmo treinamento é planejado para toda a equipe, e dessa forma, todos os atletas são submetidos à mesma carga externa de treinamento ${ }^{6}$. No entanto, a carga interna de treinamento (CIT), que consiste nas respostas fisiológicas que o organismo do atleta 
apresenta em função do estresse do treinamento é determinada pela carga externa aplicada, em conjunto com as características fisiológicas e psicológicas individuais dos atletas ${ }^{6-7}$. Uma vez que as adaptaçóes do treinamento são decorrentes da CIT, o monitoramento preciso desta variável torna-se fundamental para o sucesso do treinamento aplicado ${ }^{6,8}$.

O método da Percepção Subjetiva de Esforço da sessão (PSE-sessão; Foster ${ }^{9}$ ) tem sido proposto como uma forma prática e de baixo custo, para o monitoramento da CIT. Diversos estudos vêm apontando a validade deste método a partir da relação com outros métodos de monitoramento da CIT, particularmente aqueles baseados na frequência cardíaca, para diversas modalidades esportivas coletivas $^{6,10-12}$, inclusive no voleibol ${ }^{13}$. Outros estudos também tem revelado a validade do método da PSE-sessão através da comparação entre a dinâmica da CIT e a carga externa aplicada ${ }^{11-12}$.

Uma das vantagens da utilização do método da PSE-sessão seria a possibilidade de se quantificar a CIT em treinamentos de diferentes caracterísiticas (ex. força, resistência, técnico, etc.), permitindo dessa maneira a construção de gráficos de periodização que apresentassem distribuição das cargas de treinamento de diferentes direcionamentos ${ }^{6,8,14}$. Nesse sentido, alguns estudos tem apontado o perfil da CIT semanal aplicada em diferentes modalidades esportivas coletivas ${ }^{6,10,15}$. Por exemplo, ManzI et al. ${ }^{10}$ mostraram que as semanas sem jogos oficiais apresentam maior CIT comparadas às semanas competitivas, enquanto Wrigley et al. ${ }^{15}$ mostraram a diferença na distribuição da CIT durante

\section{Método}

\section{Amostra}

No início do período de treinamento investigado, 18 atletas pertenciam à equipe de voleibol monitorada. No entanto, dois atletas se desligaram da equipe e quatro ficaram ausentes das sessóes de treinamentos por um período superior a duas semanas por motivo de lesões e foram excluídos da amostra. Assim, a amostra do estudo foi composta por 12 atletas do sexo masculino pertencentes a uma equipe adulta que disputava a Liga Nacional de Voleibol (Liga de acesso para a Superliga de Voleibol) com média de idade de 23,5 $\pm 3,4$ anos, massa corporal de 88,2 $\pm 11,4 \mathrm{~kg}$, estatura de 190,4 $\pm 10,8 \mathrm{~cm}$ e percentual de gordura de $12,5 \pm 3,4 \%$. uma semana competitiva em função da categoria de jovens jogadores de futebol. Além da descrição de cargas semanais, MiLoski et al. ${ }^{16}$ apresentaram a distribuição das cargas de treinamento em uma temporada de futsal, mostrando diferentes magnitudes de CIT entre mesociclos do período preparatório e competitivo, ilustrando a possibilidade de se utilizar o método PSE-sessão para descrever não somente as CIT semanais, mas também o comportamento da CIT de períodos de treinamento.

Apesar da validade do método PSE-sessão para monitorar a CIT em esportes coletivos ${ }^{6,10-13,16-17}$, e de alguns estudos utilizarem esse método para monitorar a carga de treinamento no voleibol ${ }^{18-19}$, a forma como essas cargas são distribuídas durante períodos mais extensos de treinamento nessa modalidade ainda é desconhecida. O conhecimento da distribuição da CIT no voleibol se faz necessário uma vez que as demandas fisiológicas exigidas durante jogos e sessóes de treinamento ${ }^{1}$ são diferentes das exigências de outros esportes coletivos, nos quais o monitoramento longitudinal da CIT foi demonstrado em estudos anteriores $\left.{ }^{6,10,16-17}\right)$. Assim, a descrição da dinâmica da CIT durante um período de treinamento de atletas de voleibol pode enriquecer o conhecimento de envolvidos com o treinamento esportivo e auxiliar no planejamento de treinamentos futuros, bem como fornecer informaçóes de apoio para pesquisadores do esporte.

O objetivo desse estudo, portanto, foi monitorar, descrever e analisar a dinâmica da CIT obtida por meio do método da PSE-Sessão, durante um período de treinamento em atletas adultos de voleibol.
O estudo foi aprovado pelo Comitê de Ética em Pesquisa local (CEP/UFJF, parecer n. 278/2010). Todo o procedimento do teste foi esclarecido aos indivíduos que assinaram um termo de consentimento expressando sua livre participação no estudo, informando-lhes que seriam submetidos a testes classificados como sendo de risco mínimo segundo resolução 196/96 do Conselho Nacional da Saúde.

\section{Procedimentos}

O presente estudo foi realizado ao longo de um período de treinamento de 22 semanas, que antecedeu a Liga Nacional de voleibol, principal competição 
programada para a temporada da equipe. As 22 semanas foram divididas em cinco mesociclos, de acordo com os objetivos principais pré-estabelecidos pela comissão técnica da equipe para cada mesociclo, como descrito na TABELA 1. Durante esse período, os atletas participaram de jogos amistosos e de uma competição em nível estadual no terceiro mesociclo. Os atletas se apresentaram para o período de treinamento após um período de férias com duração de, aproximadamente, um mês. A carga externa de treinamento foi planejada pela comissão técnica da equipe e o monitoramento da CIT foi realizado diariamente a partir do método da PSE-sessão.

\section{Monitoramento da carga interna de treinamento}

Para monitorar a CIT foi utilizado o método da percepção subjetiva de esforço da sessão (PSEsessão) ${ }^{9}$. Trinta minutos após o término de cada sessão de treinamento, foi solicitado aos atletas que respondessem à seguinte pergunta: "Como foi seu treino?". Cada jogador classificou a intensidade da sessão de treinamento utilizando a escala de percepção subjetiva do esforço CR-10 $0^{8}$.

A CIT diária foi obtida mediante o produto do valor escolhido na escala CR-10 e a duração da sessão de treinamento em minutos ${ }^{9}$, e expressa em unidades arbitrárias (UA). Nos dias que apresentavam dois turnos de treinamento, a CIT das sessóes foi somada. Nos dias sem treinamento, foi atribuído o valor zero para a CIT diária. Além da CIT diária, a carga de treinamento semanal total (CTST) também

\section{Resultados}

As características, o direcionamento de carga, o volume de treinamento e a CIT de cada mesociclo estão descritos na TABELA 1. Ao comparar os mesociclos, pôde-se observar que o mesociclo 1 apresentou menor CTST que os mesociclos 2, 4 e $5(\mathrm{p}<0,01)$. Maiores valores de monotonia foram observados no mesociclo 4 quando comparado aos mesociclos 1,3 e 5 ( $\mathrm{p}<0,05, \mathrm{p}<0,01$ e $\mathrm{p}<$ 0,05 respectivamente) e no mesociclo 2 quando comparado ao mesociclo $3(\mathrm{p}<0,01)$. Menores foi calculada por meio do somatório das CIT diárias dos sete dias contidos em cada semana.

Para encontrar a monotonia, calculou-se a razão entre a média e o desvio padrão das CIT diárias de cada semana. Já para a obtenção do "strain", multiplicou-se a CTST pela monotonia9. Em cada mesociclo, calculou-se a média das variáveis CTST, monotonia e "Strain". A monotonia reflete a oscilação da magnitude das cargas de treinamento aplicadas nas diferentes sessóes de treinamento de um determinado período. O "strain" é uma variável que proporciona informaçóes em uma única variável a respeito do efeito da magnitude (CTST) e da distribuição (monotonia) das cargas nos jogadores. O índice elevado dessa variável pode estar associado à incidência de doenças infecciosas e lesões? .

\section{Análise estatística}

Os dados foram expressos em forma de média e desvio padrão (DP). O pressuposto de normalidade foi avaliado com o teste de Shapiro-Wilk e a esfericidade dos dados foi avaliada por meio do teste $\mathrm{M}$ de Box. Quando violada a esfericidade, utilizou-se o fator de correção Épsilon de Huynh-Feldt. Para testar a diferença entre a CTST, monotonia e "Strain" ao longo dos cinco mesociclos de treinamento, utilizouse a ANOVA de medidas repetidas, seguida por post hoc de Tukey. Calculou-se o coeficiente de variação (CV) da média da CTST das 22 semanas. Os dados foram analisados com a utilização do software SPSS (v.16, SPSS ${ }^{\circledast}$ Inc, Chicago, IL), considerando uma probabilidade de erro tipo I $(\alpha)$ de 0,05 .

valores de "Strain" foram observados no mesociclo 1 quando comparado aos mesociclos 2 e $4(\mathrm{p}<0,01)$, e no mesociclo 3 quando comparado aos mesociclos 2 e $4(\mathrm{p}<0,01)$.

A CTST das 22 semanas de treinamento está descrita na FIGURA 1. O CV da média das CTST das 22 semanas foi de $16 \%$. O valor médio da média da CTST das 22 semanas foi de 1790,9 UA. O menor valor de média da CTST das 22 semanas foi de 1324,3 UA, e o maior valor foi de 2232,7 UA. 
RF = Resistência de força;

$C A=$ Capacidade aeróbia;

CAN = Capacidade anaeróbia;

$A G=$ Agilidade;

$\mathrm{H}=$ Hipertrofia;

$\mathrm{FO}=$ Força pura;

$\mathrm{PO}=$ Potência;

$\mathrm{TT}=$ Treinamento técnico e tático;

CTST $=$ Carga de treinamento semanal total. Significativamente diferente comparado ao mesociclo 1 ("0,01; " 0,05 ); Significativamente diferente comparado ao mesociclo 2 ("0,01; \#0,05); Significativamente diferente comparado ao mesociclo $3\left({ }^{*} 0,01 ; * 0,05\right)$; Significativamente diferente comparado ao mesociclo $4\left({ }^{\dagger} 0,01 ;{ }^{\dagger} 0,05\right)$.

TABELA 1 - Descrição das características, carga externa e carga interna de treinamento dos mesociclos de treinamento da equipe de voleibol.

\begin{tabular}{lccccc}
\hline Mesociclos & 1 & 2 & 3 & 4 & 5 \\
\hline No de semanas & 6 & 5 & 4 & 4 & 3 \\
Objetivos principais & RF, TT, CA & H, AG, CAN,TT & FO, AG, TT & PO, AG, TT & TT, PO \\
No de jogos & 4 & 4 & 5 & 4 & 3 \\
Físico (min) & 112,5 & 136,0 & 105,0 & 165,0 & 135,0 \\
Técnico (min) & 117,8 & 206,8 & 125,3 & 103,5 & 103,0 \\
Tático (min) & 143,4 & 130,2 & 130,8 & 247,5 & 284,7 \\
Tempo total (min) & 373,7 & 473 & 361,1 & 516,0 & 522,7 \\
CTST (UA) & $1509,2 \pm 329,4$ & $1987,1 \pm 371,5^{*}$ & $1724,6 \pm 429,1$ & $1956,5 \pm 269,9^{*}$ & $1894,9 \pm 415,8^{*}$ \\
Monotonia (UA) & $1,2 \pm 0,3$ & $1,3 \pm 0,2$ & $1,1 \pm 0,2^{*}$ & $1,4 \pm 0,1^{* * *}$ & $1,2 \pm 0,2^{+}$ \\
"Strain" (UA) & $1929,4 \pm 630,6$ & $2758,2 \pm 822,5^{*}$ & $2029,9 \pm 736,7^{*}$ & $2802,7 \pm 580,7^{* *}$ & $2369,1 \pm 762,1$ \\
\hline
\end{tabular}

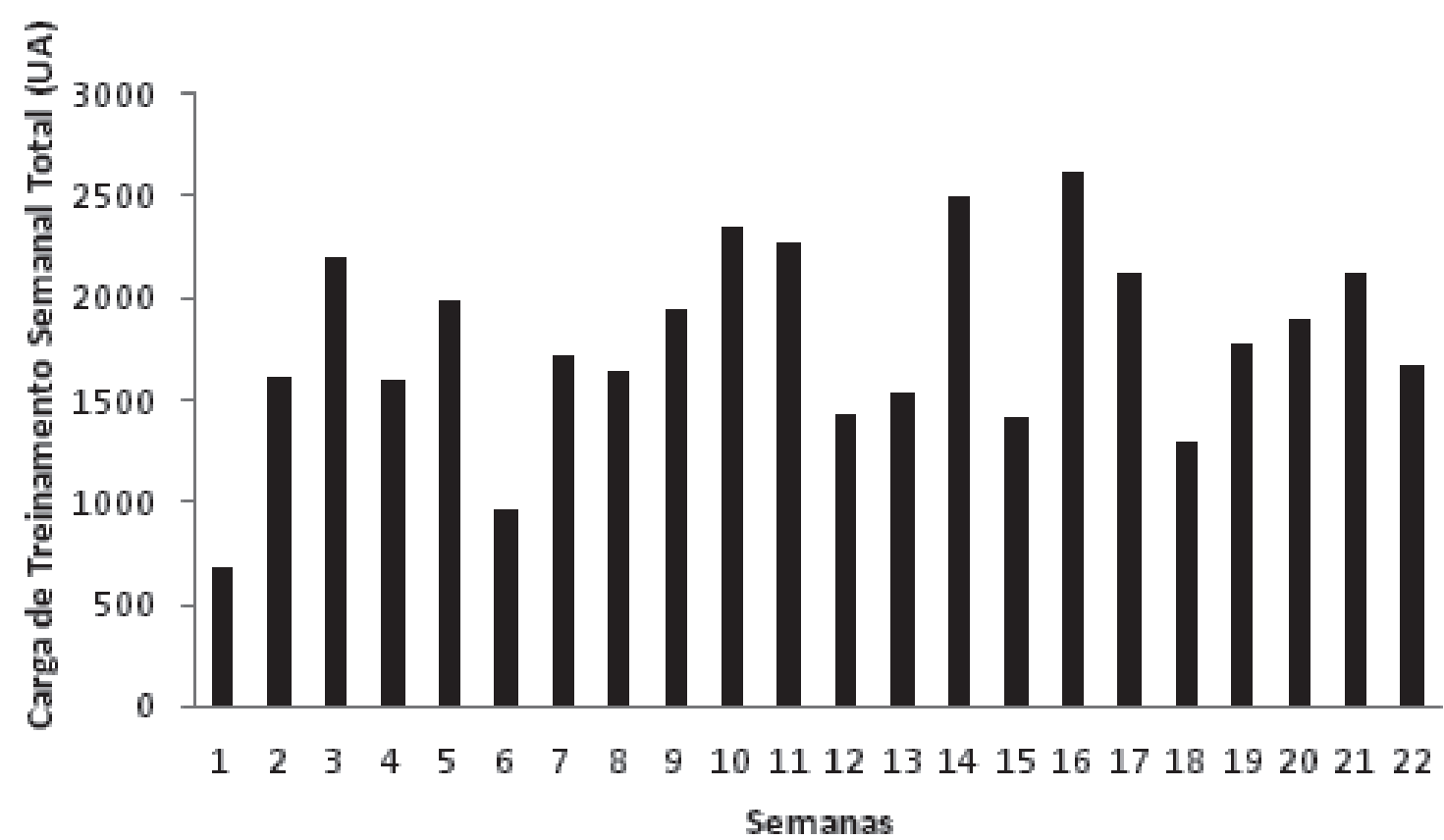

FIGURA 1 - Descrição da carga de treinamento semanal total das 22 semanas de treinamento.

\section{Discussão}

Os principais achados deste estudo foram que a CTST aumentou do primeiro para o segundo mesociclo e houve manutençáo durante os demais mesociclos investigados. Além disso, foram descritas CTST de maior magnitude intercaladas com de menor magnitude durante as 22 semanas. Também foram observados baixos valores de monotonia e de "Strain" durante o período de treinamento investigado.

Os resultados da presente investigação demonstram que o longo período de treinamento que antecedeu a competição principal programada para a equipe permitiu que a comissão técnica planejasse o treinamento com progressão das cargas do primeiro para o segundo mesociclo, e manutenção das mesmas até o final do período de treinamento descrito, que consistia na semana anterior aos jogos da Liga Nacional de Voleibol.

Entretanto, o comportamento da CIT apresentado por uma equipe de futsal ${ }^{17}$ foi diferente do descrito no presente estudo. No estudo supracitado, a carga de treinamento foi alta no mesociclo inicial 
$(-2900$ UA) e diminuiu ao aproximar do período competitivo $(\sim 1600 \mathrm{UA})$. A diferença na dinâmica da CIT mostrada na presente investigação pode estar associada à maior duração do período de treinamento analisado, que se apresentou como um longo período de preparação para a competiçáo alvo, que por sua vez, permitiria a escolha de uma estratégia de periodização do treinamento diferente. O comportamento apresentado pelas CTST das 22 semanas de treinamento, o qual intercala momentos de maior e menor magnitude é coerente com a dinâmica de distribuição da carga de treinamento proposta por alguns modelos de periodização, principalmente o modelo de periodização tradicional ${ }^{20}$.

Os valores médios de CTST apresentados nos mesociclos do presente estudo estão entre 1509 e 1987 UA (semana com menor CTST com 686 UA e semana com maior CTST com 2620 UA). Valores de outros estudos com esportes coletivos se aproximam aos valores apresentados no presente estudo como, por exemplo: em atletas de futsal, MiLoski et al. ${ }^{16}$ apresentaram mesociclos com valores médios de CTST entre 1170 e 2509 UA; em atletas de "rugby", Coutts et al. ${ }^{21}$ apontam valores de CTST entre 1238 e 3107 UA; também em atletas de "rugby", CoutTs et al. ${ }^{22}$ apontam valores de CTST entre 1387 e 3296 UA; já em atletas de basquetebol, MANZI et al..$^{10}$ apresentaram valores mais elevados, entre 2791 e 3334 UA.

Apesar da proximidade dos valores de CTST dos estudos supracitados sugerirem haver uma tendência de magnitudes de CTST em esportes coletivos, a dinâmica da distribuição das CITs descritas se difere de acordo com a modalidade esportiva. Além disso, a característica de cada modalidade, as quais exigem diferentes demandas fisiológicas, pode ser um fator determinante para as diferenças nas magnitudes de carga de treinamento apresentada por cada modalidade. Por exemplo, o "rugby" é um esporte de contato, intermitente, no qual intercala momentos de alta intensidade (corrida em alta intensidade, sprints e colisóes) com momentos de baixa intensidade (andar, trotar, ficar parado ${ }^{23}$. De maneira diferente, o voleibol é uma modalidade esportiva intermitente, no qual intercala momentos jogados com duraçáo média aproximada de 11 segundos com intervalos de recuperação médios aproximados de 14 segundos, sem contato físico com outros jogadores, e com os saltos e deslocamentos laterais curtos como as principais açôes executadas nos momentos joga$\operatorname{dos}^{1}$. Nesse contexto, a maior demanda fisiológica exigida pelo "rugby" jusfica os maiores valores de CIT apresentadas nos estudos supracitados quando comparados aos valores descritos no presente estudo com atletas de voleibol.

O resultado do presente estudo em conjunto com os de investigaçóes prévias ${ }^{16-17,24}$ salientam que a diferença na dinâmica da CIT demonstrada nos mesmos, justifica a necessidade do monitoramento da CIT em diferentes modalidades esportivas e em diferentes realidades competitivas. Dessa forma, entende-se que os modelos de periodização de treinamento não devem ser copiados de modelos previamente executados e sim, formulados de acordo com a realidade apresentada, dependendo de inúmeros fatores como, por exemplo, tempo para preparação, calendário competitivo e nível de qualificação da equipe ${ }^{25}$.

Um período de treinamento com duração de 22 semanas objetivando uma competição principal, como o que foi relatado no presente estudo é considerado demasiadamente longo para a realidade contemporânea dos esportes coletivos ${ }^{26}$, fato que pode ajudar a explicar a utilizaçáo de um programa de treinamento que aproxime dos conceitos dos modelos tradicionais de periodizaçáo. No caso do presente estudo, a competição alvo, Liga Nacional de Voleibol, se apresentou como uma competição de curta duração, dividida em fase classificatória (três jogos realizados em dias consecutivos) e fase final (cinco jogos realizados em dias consecutivos) o que permitiu programar um período relativamente longo para a preparação dos atletas. No entanto, a realidade de outros esportes coletivos ${ }^{20,26} \mathrm{e}$ até mesmo, outras competiçóes no voleibol nacional, como a Superliga Masculina de Voleibol, apresentam um longo período competitivo.

O método da PSE-sessão, é o produto entre a intensidade e o volume de treinamento em uma única variável $^{8}$. Desse modo, os mesociclos com maior volume de treinamento, como os mesociclos 4 e 5 também apresentam magnitudes de CTST elevadas comparado com os outros mesociclos. Nota-se que nesses mesociclos (4 e 5), destinou-se um maior tempo para o treinamento tático e o objetivo principal do treinamento físico foi desenvolver capacidades específicas ao voleibol, tendo em vista a proximidade da competiçâao alvo. Por outro lado, o mesociclo 3, momento no qual utilizou-se jogos de um campeonato como preparação para a competição alvo, o menor volume de treinamento refletiu em menores valores de CTST.

Os mesociclos do período preparatório apresentaram monotonia abaixo de 2,0 UA. Valores de monotonia acima de 2,0 UA são considerados reflexos de pouca oscilação das cargas de treinamento, o que favorece as adaptaçóes negativas do treinamento (queda do desempenho, aumento de incidência de doenças 
infecciosas e lesôes ${ }^{9}$. Dessa maneira, os valores de monotonia observados nesse estudo demonstram que as cargas foram bem distribuídas durante as semanas de treinamento, intercalando dias com maior e menor magnitude de cargas. Valores baixos de monotonia foram demonstrados em estudos anteriores com atletas de ciclismo ${ }^{27}$, "rugby" ${ }^{22}$, futsal ${ }^{16}$ e velocistas de 400 metros $^{28}$. Essa característica de distribuição de cargas permite que os atletas se recuperem de dias com cargas de maior magnitude e consequentemente melhorem o desempenho, bem como evita o acúmulo de fadiga que pode ocasionar adaptaçóes indesejadas (queda do desempenho, lesóes, infecção do trato respiratório superior) derivadas do treinamento ${ }^{20}$.

Como a monotonia esteve controlada por todo período de treinamento, os valores de "strain" descritos no presente estudo apresentaram uma dinâmica próxima aos da CTST, exceto para o mesociclo 3 , no qual os valores de "strain" foram menores que os valores dos mesociclos 2 e 4 . O maior número de jogos no mesociclo 3 pode justificar a menor magnitude de CTST e monotonia, logo, o menor valor de "strain" nesse mesociclo. Miloski et al. ${ }^{16}$ demonstraram que os mesociclos com maior número de jogos de futsal são aqueles nos quais é aplicado um maior número de sessóes regenerativas quando comparados aos mesociclos com número reduzido de jogos.

O coeficiente de variaçáo de $16 \%$ das CTST das 22 semanas sugere que diferentes atletas podem perceber a carga externa planejada para toda equipe de maneira diferente, refletindo em diferentes valores de $\mathrm{CIT}^{4,6}$. Os valores extremos (menor valor de 1324,3 UA e o maior valor de 2232,7 UA) demonstram que em uma mesma equipe, alguns atletas podem apresentar valores de CIT bem diferentes. A capacidade física ${ }^{7} \mathrm{e}$ a função do atleta (posição) na equipe ${ }^{1}$ são fatores que podem influenciar na dinâmica da CIT. Apesar desses valores náo serem muito diferentes dos valores médios da equipe (1790,9 UA), os valores extremos apresentados por alguns atletas, se diferenciam de maneira considerável. Esses resultados sugerem que a análise da média das CIT da equipe proporciona informaçóes relevantes do comportamento dessa variável ao longo do treinamento. No entanto, uma análise individual se faz necessária devido à discrepância de valores de CIT observados por alguns jogadores.

Podemos assumir como uma limitação do presente estudo o uso de apenas uma equipe de voleibol como a amostra participante. Desse modo, o estudo descreve a dinâmica da carga de treinamento de apenas uma equipe, e não necessariamente a dinâmica da CIT de equipes de voleibol durante um período de treinamento. Isso sugere a necessidade de outros estudos, descrevendo a CIT de outras equipes de mesmo nível competitivo, bem como estudos com um número maior de equipes, para assim, promover uma análise mais acurada sobre a dinâmica da CIT de equipes de voleibol durante um período de treinamento que antecede uma competição alvo.

Conclui-se que a dinâmica da CIT de jogadores de voleibol monitorada por meio do método PSE-sessáo durante as 22 semanas de treinamento apresentou semanas com carga de maior magnitude intercalada com semanas com carga de menor magnitude. A CIT aumentou do primeiro para o segundo mesociclo com manutençấo da mesma nos mesociclos seguintes.

\begin{abstract}
Internal training load monitoring across a period training in volleyball players

The aim of this study was to monitor and analyze the dynamics of internal training load using session rating of perceived exertion (session-RPE) method during a training period of volleyball. Twelve volleyball players $(23.5 \pm 3.4 \mathrm{y}, 88.2 \pm 11.4 \mathrm{~kg}, 190.4 \pm 10.8 \mathrm{~cm})$ were monitored during 22 training weeks divided into five mesocycles. The ANOVA revealed that the total week training load (TWTL), monotony, and strain were different among the mesocycles $(p<0.05)$. The higher values of TWTL were showed during mesocycles 2,4 , and 5 . Monotony presented values below $1.4 \pm 0.1 \mathrm{AU}$. The mean TWTL of the 22 weeks presented a mean of $1790.9 \mathrm{UA}$ and a coefficient of variation of $16 \%$. The lower and higher mean values of TWTL of the 22 weeks were 1324.3 and $2232.7 \mathrm{UA}$, respectively. Thus, the internal training load distribution presented moments with higher and lower magnitude between weeks, and increase from 1st to 2 nd mesocycle with maintenance of this following others mesocycles.
\end{abstract}

KEY WoRDS: Sport; Physical education; Training; Performance. 


\section{Referências}

1. Sheppard JM, Gabbett T, Taylor KL, Dorman J, Lebedew AJ, Borgeaud R. Development of a repeated-effort test for elite men's volleyball. Int J Sports Physiol Perform. 2007;2:292-304.

2. Peńa J, Guerra JR, Buscà B, Serra N. Which skills and factors better predict winning and losing in high-level men's volleyball? J Strength Cond Res. 2013;27:487-93.

3. Meeusen R, Duclos M, Gleeson M, Rietjens G, Steinacker J, Urhausen A. Prevention, diagnosis and treatment of the Overtraining Syndrome - ECSS Position Statement 'Task Force'. Eur J Sport Sci. 2006;6:1-14.

4. Borresen J, Lambert MI. The quantification of training load, the training response and the effect on performance. Sports Med. 2009;39:779-95.

5. Nesser TW, Demchak TJ. Variations of preseason conditioning on volleyball performance. J Exerc Physiol . 2007;10:35-42.

6. Impellizzeri FM, Rampinini E, Coutts AJ, Sassi A, Marcora SM. Use of RPE-based training load in soccer. Med Sci Sports Exerc. 2004;36:1042-7.

7. Milanez VF, Pedro RE, Moreira A, Boullosa DA, Salle-Neto F, Nakamura FY. The role of aerobic fitness on session rating of perceived exertion in futsal players. Int J Sports Physiol Perform. 2011;6:358-66.

8. Foster C, Florhaug JA, Franklin J, et al. A new approach to monitoring exercise training. J Strength Cond Res. 2001;15:109-15.

9. Foster C. Monitoring training in athletes with reference to overtraining syndrome. Med Sci Sports Exerc. 1998;30:1164-8.

10. Manzi V, D’Ottavio S, Impellizzeri FM, Chaouachi A, Chamari K, Castagna C. Profile of weekly training load in elite male professional basketball players. J Strength Cond Res. 2010;24:1399-406.

11. Casamichana D, Castellano J, Calleja J, Roman JS, Castagna C. Relationship between indicators of training load in soccer players. J Strength Cond Res. 2013;27:369-74.

12. Scott TJ, Black C, Quinn J, Coutts AJ. Validity and reliability of the session RPE method for quantifying training in Australian football: a comparison of the CR10 and CR100 scales. J Strength Cond Res. 2013;27:270-6.

13. Bara Filho MG, Andrade FC, Nogueira RA, Nakamura FY. Comparison of different methods of internal load control in volleyball players. Rev Bras Med Esporte. 2013;19:143-46.

14. Nakamura FY, Moreira A, Aoki MS. Training load monitoring: is the session rating of perceived exertion a reliable method? Rev Educ Fís/UEM. 2010;21:11.

15. Wrigley R, Drust B, Stratton G, Scott M, Gregson W. Quantification of the typical weekly in-season training load in elite junior soccer players. J Sports Sci. 2012;30:1573-80.

16. Miloski B, Freitas VH, Bara-Filho MG. Monitoring of the internal training load in futsal players over a season. Rev Bras Cineantropom Desempenho Hum. 2012;14:671-79.

17. Freitas VH, Miloski B, Bara-Filho MG. Quantification of training load using session RPE method and performance in futsal. Rev Bras Cineantropom Desempenho Hum. 2012;14:73-82.

18. Moreira A, Freitas CG, Nakamura FY, Aoki MS. Session RPE and stress tolerance in young volleyball and basketball players. Rev Bras Cineantropom Desempenho Hum. 2010;12:345-51.

19. Dias R, Frollini AB, Brunelli DT, et al. Immune parameters, symptoms of upper respiratory tract infections, and training-load indicators in volleyball athletes. Int J Gen Med. 2011;4:837-44.

20. Issurin VB. New horizons for the methodology and physiology of training periodization. Sports Med. 2010;40:189-206.

21. Coutts AJ, Reaburn P, Piva TJ, Rowsell GJ. Monitoring for overreaching in rugby league players. Eur J Appl Physiol. 2007;99:313-24.

22. Coutts A, Reaburn P, Piva TJ, Murphy A. Changes in selected biochemical, muscular strength, power, and endurance measures during deliberate overreaching and tapering in rugby league players. Int J Sports Med. 2007;28:116-24.

23. Waldron M, Twist G, Highton J, Worsfold P, Daniels M. Movement and physiological match demands of elite rugby league using portable global positioning systems. J Sports Sci. 2011;29:1223-30.

24. Gabbett TJ. Performance changes following a field conditioning program in junior and senior rugby league players. J Strength Cond Res. 2006;20:215-21.

25. Kiely J. Periodization paradigms in the 21st century: evidence-led or tradition-driven? Int J Sports Physiol Perform. 2012;7:242-50.

26. Moreira A. The training periodization and the emerged questions: the team sports' case. Revista Andaluza de Medicina del Deporte. 2010;3:170-8.

27. Delattre E, Garcin M, Mille-Hamard L, Billat V. Objective and subjective analysis of the training content in young cyclists. Appl Physiol Nutr Metab. 2006;31:118-25.

Rev Bras Educ Fís Esporte, (São Paulo) 2015 Jan-Mar; 29(1):5-12 • 11 
Freitas VH, et al.

28. Suzuki S, Sato T, Maeda A, Takahashi Y. Program design based on a mathematical model using rating of perceived exertion for an elite Japanese sprinter: a case study. J Strength Cond Res. 2006;20:36-42.

ENDEREÇO

Maurício Gattás Bara Filho

Faculdade de Educação Física e Desportos

Universidade Federal de Juiz de Fora

R. José Lourenço Kelmer, s/n

36036-330 - Juiz de Fora - MG - BRASIL

e-mail:mgbara@terra.com.br

Recebido para publicação: 04/12/2012

1a. revisão: 06/04/2013

2a. revisão: 08/08/2013

3a. revisão: 26/12/2013

Aceito: 20/03/2014

12 • Rev Bras Educ Fís Esporte, (São Paulo) 2015 Jan-Mar; 29(1):5-12 